\title{
A Survey of Semantic Construction and Application of Satellite Remote Sensing Images and Data
}

\author{
Hui Lu, Nanjing University of Information Science and Technology, Nanjing, China \\ Qi Liu, Nanjing University of Information Science and Technology, Nanjing, China \\ Xiaodong Liu, Edinburgh Napier University, Edinburgh, UK \\ Yonghong Zhang, Nanjing University of Information Science and Technology, Nanjing, China
}

\begin{abstract}
With the rapid development of satellite technology, remote sensing data has entered the era of big data, and the intelligent processing of remote sensing images has been paid more attention. Through the semantic research of remote sensing data, the processing ability of remote sensing data is greatly improved. This paper aims to introduce and analyze the research and application progress of remote sensing image satellite data processing from the perspective of semantics. Firstly, it introduces the characteristics and semantic knowledge of remote sensing big data. Secondly, the semantic concept, semantic construction, and application fields are introduced in detail. Then, for remote sensing big data, the technical progress in the study field of semantic construction is analyzed from four aspects-semantic description and understanding, semantic segmentation, semantic classification, and semantic search-focusing on deep learning technology. Finally, the problems and challenges in the four aspects are discussed in detail in order to find more directions to explore.
\end{abstract}

\section{KEYWORDS}

Automatic Analysis, Deep Learning, Remote Sensing, Satellite Remote Sensing, Semantic Construction, Semantic Knowledge

\section{INTRODUCTION}

More and more countries have launched a series of remote sensing satellites, and satellite remote sensing technology has developed rapidly. These satellites include land satellites, meteorological satellites, synthetic aperture radar, etc., covering infrared, visible and multispectral bands. Through the long-term continuous Earth Observation of satellites in different return visit periods, a large number of remote sensing earth observation data with multi spectral, multi temporal, multi spatial and multi type resolution have been accumulated for researchers (J. Zhu et al., 2016). Remote sensing data has the characteristics of huge capacity, fast efficiency, diverse types, rich value and difficult identification. Its characteristics are shown in Figure 1, indicating that remote sensing has entered the era of big data.

These remote sensing data come from different data sources and have multiple feature dimensions, which respect the changes of land surface information in spatial, temporal and spectral dimensions.

DOI: 10.4018/JOEUC.20211101.oa6

This article published as an Open Access article distributed under the terms of the Creative Commons Attribution License (http://creativecommons.org/licenses/by/4.0/) which permits unrestricted use, distribution, and production in any medium, provided the author of the original work and original publication source are properly credited. 


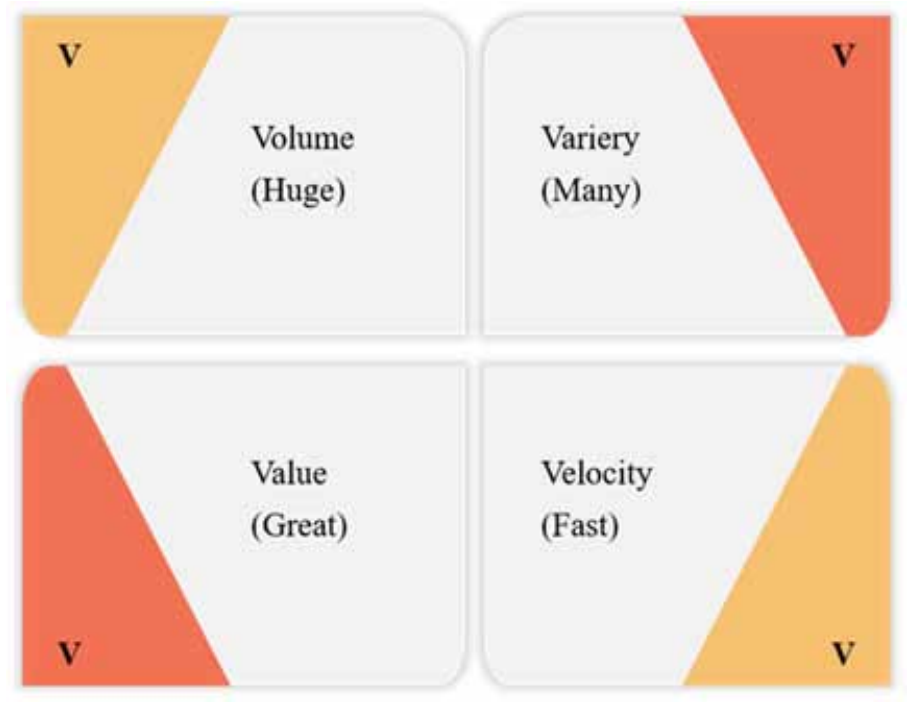

For example, the current landsat series of satellite sensors have accumulated a large number of surface observation data through more than 40 years of long-term observation (phiri \& morgenroth, 2017). Modis sensors provide global observation data for about 17 years (xiong et al., 2017). In addition, more and more remote sensing satellites provide huge remote sensing satellite data. Table 1 lists the features of satellite data commonly used by current researchers. Under the background of the internet of things (iot) era, information technology is changing with each passing day, especially the big data processing and analysis technology of the internet of things, which has achieved good results in the methods of distributed tensor sequence decomposition, visual feature recognition, datadriven and edge server quantification, which has achieved well results in the methods of distributed tensor sequence decomposition, visual feature recognition, data-driven and edge server quantification and has been well applied to industrial intelligence, gradually realizing industrial intelligence and constantly changing all aspects of industrial manufacturing (ren et al., 2020; x. Wang, yang, song, et al., 2020; x. Wang, yang, wang, ren and deen, 2020; xu et al., 2020). Inevitably, it also promotes the rapid development of remote sensing big data technology. Great changes have taken place in the research and application of semantic construction of remote sensing big data.

Semantics is the meaning of a word, a sentence or a paragraph. Human beings make corresponding judgments or actions by understanding semantics. However, the computer is only regarded as a code segment composed of " 0 " and " 1 ", and it cannot understand the meaning contained in the rich and extensive information. In order to be able to introduce the meaning of information into the meaning of the computer. This idea was first put forward by tim berners ley, an american. In his article "the road to the semantic web", he pointed out: "the semantic web is the distribution center of information. In addition to the communication between people, computers can also participate in it to help people complete the corresponding things together". Therefore, the semantic technologies are introduced into the computer, so that the computer also has ability to read information semantics like human. A new development of semantic world is "block", which is a mixture of rdf and attribute mapping. It is inspired by cognitive science. It focuses on machine learning, integrates graphics and statistics, and provides a new model for artificial intelligence that can simulate the human brain at a higher level than a single neuron. The introduction of semantics into remote sensing data processing will greatly promote understanding and application of remote sensing data knowledge, and play an irreplaceable role in remote sensing field. This paper focuses on the research progress of semantic-based remote 
sensing data processing technology, mainly to investigate the associated technologies of semantic construction of remote sensing data, and discuss the research, put forward problems and challenges, hoping to provide reference value for researchers in related fields.

The rest of this paper is divided into several parts: semantic concept and semantic construction methods have been introduced in 2 section; followed by semantic construction and application of satellite remote sensing data from four aspects in 3 section; the challenges and prospects of current semantic construction and application fields have been discussed in 4 section with conclusion in 5 section.

\section{SEMANTICS}

Semantic technologies transform data processing into semantic knowledge processing, which brings efficient and powerful knowledge-based system, and have gradually penetrated into various fields and plays a vital role in scientific and technological study. This section analyzes the semantic concept and semantic construction and common semantic application fields.

\subsection{Semantics Concept and Construction}

The data itself is meaningless. However, only using meaningful data. Specifically, when data is changed into information, the meaning of data representation is semantics. Semantics represents the concrete conceptual meaning and the relationship between the objects corresponding to data in the real world. More precisely, it is the interpretation and logical representation of data in a certain field. In computer science, semantics usually refers to the interpretation of computer symbols that describe the real world. It is a concept that computers and the real world are interrelated.

Semantic construction refers to the process of semantic representation construction of natural language expression (Gardent \& Kallmeyer, 2003). From the programmer's point of view, it is to study the method of automating this process. In the computer field, the current research mainly focuses on semantic description, semantic classification methods, semantic knowledge base, semantic map, semantic layer interpretation strategy and the construction of knowledge semantic model under the Semantic Web (Collarana et al., 2017; Liu et al., 2018; S. Wang et al., 2018). There are many semantic description languages, and the main semantic description languages recommended by $\mathrm{W} 3 \mathrm{C}$ are RDF, RDFS and OWL. Besides, the common tool for semantic construction is protege. It provides a graphical and interactive knowledge ontology development environment, and supports ontology languages such as RDF, RDFS, OWL to edit and modify ontology outside the system. In addition, Neon, OilEd, MediaWiki and OntoWiki are also used as semantic building tools.

\subsection{Semantic Application Fields}

Semantics can be applied in many fields of today's society, and they are widely used. This section focuses on the application of semantics in semantic web, agriculture, aerospace, remote sensing big data.

\subsubsection{Applications of Semantics in Semantic Web}

As an extension of the present web, semantic web is developing rapidly. With good knowledge expression, communication, sharing and reasoning ability, it has been widely recognized in many industries and fields. In short, semantic web exists mainly to understand human language. It can make the communication between people and computers very easy. The great idea originally proposed by Tim bemers. LEC. By showing the syntax structure and semantics of web content in the form of knowledge representation, in order to realize the sharing with other information sources, people, machines and machines can accurately understand each other, so as to achieve the maximum degree of interoperability. If the semantic web can work smoothly, the computer should be able to organize information and rule sets in a structured way, so that the computer can use the rules for automatic 
Table 1. Common Remote Sensing Satellite Data

\begin{tabular}{|c|c|c|c|c|c|c|c|}
\hline No & \multicolumn{2}{|l|}{ Name } & Country & \begin{tabular}{|l} 
Launch \\
Time \\
\end{tabular} & Spectral Band & Spatial Resolution & \begin{tabular}{|l|}
$\begin{array}{l}\text { Resolution } \\
\text { Level }\end{array}$ \\
\end{tabular} \\
\hline 1 & \multicolumn{2}{|c|}{ Landsat8 } & America & 2013 & 11 bands & $15 / 30 / 100$ & middle \\
\hline 2 & \multicolumn{2}{|c|}{ MODIS } & America & I & 36 bands & $250 / 500 / 1000$ & low \\
\hline 3 & \multicolumn{2}{|c|}{ NOAA series } & America & / & $\begin{array}{l}\text { Red, near } \\
\text { infrared, mid } \\
\text { infrared and } \\
\text { two thermal } \\
\text { infrared }\end{array}$ & 1100 & 1 \\
\hline \multirow[t]{3}{*}{4} & \multirow{3}{*}{$\begin{array}{l}\text { SPOT } \\
\text { series }\end{array}$} & SPOT4 & France & 1999 & $\begin{array}{l}\text { Red, green, } \\
\text { blue, mid far } \\
\text { infrared }\end{array}$ & $\begin{array}{l}\text { panchromatic: } \\
10\end{array}$ & \multirow{3}{*}{ high } \\
\hline & & SPOT5 & France & 2001 & $\begin{array}{l}\text { Red, green, } \\
\text { blue, mid far } \\
\text { infrared }\end{array}$ & $\begin{array}{l}\text { panchromatic: } \\
2.5 / 5\end{array}$ & \\
\hline & & SPOT6/7 & France & $2013 / 2014$ & $\begin{array}{l}\text { Red, green, } \\
\text { blue, near } \\
\text { infrared }\end{array}$ & $\begin{array}{l}\text { panchromatic: } \\
1.5\end{array}$ & \\
\hline 5 & \multicolumn{2}{|c|}{ Sentienl-2A } & ESA & 2015 & $\begin{array}{l}\text { There are } 13 \\
\text { bands of visible } \\
\text { light, short } \\
\text { wave, infrared. } \\
\text { etc }\end{array}$ & $10 / 20 / 60$ & 1 \\
\hline 6 & \multicolumn{2}{|l|}{ FY } & China & I & $\begin{array}{l}\text { Visible light, } \\
\text { near infrared, } \\
\text { mid far infrared, } \\
\text { thermal infrared }\end{array}$ & 1100 & low \\
\hline 7 & \multicolumn{2}{|c|}{$\mathrm{HJ}-1 \mathrm{~A} / \mathrm{B}$} & China & 2008 & $\begin{array}{l}\text { Multispectral } \\
\text { near mid } \\
\text { infrared } \\
\text { (4-band), } \\
\text { hyperspectral } \\
\text { (111 band) }\end{array}$ & $30 / 100$ & 1 \\
\hline \multirow{4}{*}{8} & \multirow{4}{*}{$\begin{array}{l}\text { GF } \\
\text { series }\end{array}$} & GF-1 & \multirow{4}{*}{ China } & 2013 & RGB+NIR & $2 / 8 / 16$ & \multirow{4}{*}{ high } \\
\hline & & GF-2 & & 2014 & RGB+NIR & 1 & \\
\hline & & GF-3 & & 2015 & \begin{tabular}{|l} 
SAR \\
(C band)
\end{tabular} & $1 \sim 500$ & \\
\hline & & GF-4 & & 2016 & $\begin{array}{l}\text { Visible light, } \\
\text { near infrared, } \\
\text { medium wave } \\
\text { infrared }\end{array}$ & $50 / 400$ & \\
\hline \multirow{2}{*}{9} & \multirow{2}{*}{$\begin{array}{l}\mathrm{ZY} \\
\text { series }\end{array}$} & $\begin{array}{l}\mathrm{ZY}-1 \\
02 \mathrm{C}\end{array}$ & \multirow{2}{*}{ China } & 2011 & $\begin{array}{l}\text { Red, green, } \\
\text { near infrared }\end{array}$ & $5 / 10 / 2.36$ & 1 \\
\hline & & ZY-3 & & 2012 & RGB+NIR & $2.1 / 3.5 / 6$ & high \\
\hline
\end{tabular}


reasoning. The semantic web architecture is a seven-tier model. Semantic Web has brought a lot of new development opportunities, which has a very extensive range of applications. Such as knowledge management, semantic search, semantic web services, semantic grid, P2P and so on. XML, RDF, RDF schema and ontology are common key technologies. Based on Se mantic Web technology, various applications can be developed (Khamparia \& Pandey, 2017).

Klusch et al. elaborated many research fields of semantic web technology application and the specific role of semantic technologies (Klusch et al., 2016). Pauwels and others introduced the application of semantic web technology in architecture fields, engineering and construction, and proposed the viewpoint of overcoming the operation problems of interdisciplinary software tools, improving the process of information exchange, connecting applications in different fields, and realizing resource mining and expansion (Pauwels, Zhang, \& Lee, 2017). Zhong et al. introduced the research progress of semantic web related services in detail, and proposed key technologies. Such as knowledge management, semantic search and semantic web services (Zhong et al., 2009). Yuan et al. elaborated the applications and development of semantic technologies in the Internet of Things, and analyzed the semantic requirements of all levels of the Internet of Things (Yuan et al., 2014). This paper mainly elaborates and analyzes the applications of semantic technologies in IoT resource description and modeling, perceptual data description and processing, perceptual data semantic annotation, service construction and provision, data storage and query. It is also pointed out that the applications of semantic technologies will improve the collaborative ability among IoT resources, information model, data providers and consumers, and will be conducive to data acquisition and integration, resource discovery, semantic reasoning and knowledge extraction. Currently, the domestic study on Semantic Web is yet in the initial phase, which needs to be further explored.

\subsubsection{Applications of Semantics in Agriculture}

Agricultural information technology is a major driving force to realize the high-speed, healthy and sustainable development of agriculture. It is a new frontier application discipline and a new field generated by the cross penetration of agricultural science and information science. The applications of semantic technologies in agriculture is also pervasive, such as semantic based web service technology, agricultural information semantic retrieval technology, agricultural data semantic association technology and so on. Chen et al. proposed a new agricultural information semantic retrieval model based on dual semantic space. It is a retrieval model based on improved random index semantic space and potential semantic space. The model improves the semantic retrieval performance of large-scale agricultural information and is applied to large-scale agricultural information retrieval (Y. Chen et al., 2012). Jadhav et al. proposed an automatic semantic segmentation and classification method based on agricultural remote sensing data, which will not lose a lot of data. A Particle Swarm Optimization (PSO) algorithm and depth residual network are proposed to achieve good crop classification (Jadhav \& Singh, 2018). Garcia et al. put forward the agricultural knowledge management platform, which aims to conduct unified semantic information search for agricultural knowledge, realize knowledge storage and sharing, help users search faster and contribute to agricultural knowledge search (Garcia et al., 2019).

\subsubsection{Applications of Semantics in Aerospace}

The rapid progress of information technology, a great deal of data information is produced in the aerospace field, and semantic-based data processing technology has been widely applied. In the knowledge system of aerospace domain, the most commonly used strategy for semantic analysis of aerial images is ontology reasoning strategy based on knowledge driven semantic rules. Kasimov et al. proposed an applied semantic model of aerial image action and resource. The image is analyzed from the semantic point of view. The structure of aerial image is described more intuitively, and the aerial image is described based on content. The target detection is realized (Kasimov et al., 2019). Dai et al proposed an ontology-based knowledge network model construction method for aerospace 
product development. Ontology description was used to realize the uniqueness of knowledge model description and knowledge understanding, and knowledge network was established. Virtual prototype, development tasks and aerospace terms were introduced into knowledge network model, and its knowledge system was analyzed and applied in practice (DAI et al., 2018). Taymaz et al. applied knowledge semantic tools to parts management of aerospace products, proposed a semantic based aerospace parts search engine, established a standard part data model, used Resource Description Framework (RDF) for data model, queried standard parts from RDF repository, and found link relationships. However, the prototype system is still in its infancy and needs to be improved and optimized (Taymaz et al., 2013). Mahmood et al. proposed to use semantic knowledge for automatic traceability chain recovery in aerospace demand supervisory system. The purpose is to cut down the consumption cost of time and resources, help software engineering projects meet business needs, and improve the efficiency of time management (Mahmood et al., 2015).

\subsubsection{Applications of Semantics in Remote Sensing Big Data}

Remote sensing big data is widely used in agriculture, industry, disaster emergency management, environmental and ecological monitoring, meteorological prediction, road extraction and even military fields. Among them, for the semantic application of remote sensing big data is also embodied in all aspects of the above fields, the remote sensing big data is used for the processing and analysis of multisource, multi-band and multi-temporal spatial information. Through semantic analysis, the researchers can understand land cover change and urban expansion change. For example, the observation data of Landsat long time series are applied to forest change detection (Kim et al., 2016), the application of Fengyun series meteorological satellites in grassland fire monitoring (Jiang et al., 2018), and the multi-level semantic scene change interpretation strategy of remote sensing images to describe the transition from forest degradation to urban expansion (Ghazouani et al., 2019). Besides, with the rise of semantic-based multi-source remote sensing data processing technology, it brings new opportunities and challenges for the storage, organization, analysis and processing of remote sensing data.

\section{SEMANTIC APPLICATION IN SATELLITE REMOTE SENSING}

In the field of semantics, semantic construction has always been the focus of attention. Polysemy exists in any language. How to distribute and expand multiple meanings in the same expression is the focus of semantic construction. In this paper, it mainly describes how to express, understand and describe semantics in the field of information technology. Furthermore, it summarizes the specific division of labor and related application technologies of current remote sensing data semantic construction. As shown in Figure 2-Figure 5, they are mainly divided into semantic understanding and description, semantic segmentation, semantic classification and semantic retrieval separately, focusing on its research in remote sensing big data. Table 2 summarizes the commonly used data sets according to the four types of remote sensing semantic research introduced in this paper. These data sets are applied for the semantic research of remote sensing data. Obviously, the data sets in the table are not comprehensive, but they can provide reference for researchers.

\subsection{Research and Application of Remote Sensing Semantic Understanding and Description}

The semantic understanding and description of remote sensing image mainly refers to the process of using machine learning, statistical learning, data mining and other methods to describe the satellite image by using the features of high space, high spectrum and high radiation resolution. Figure 6 describes its basic concept and illustrates its specific meaning with a simple example (Qu et al., 2016). Remote sensing image semantic understanding has a hierarchical structure with three levels, corresponding to low-level visual features, intermediate semantic features and high-level sampling semantics. The low-level and high-level form a closed-loop from data-driven to knowledge-driven, 


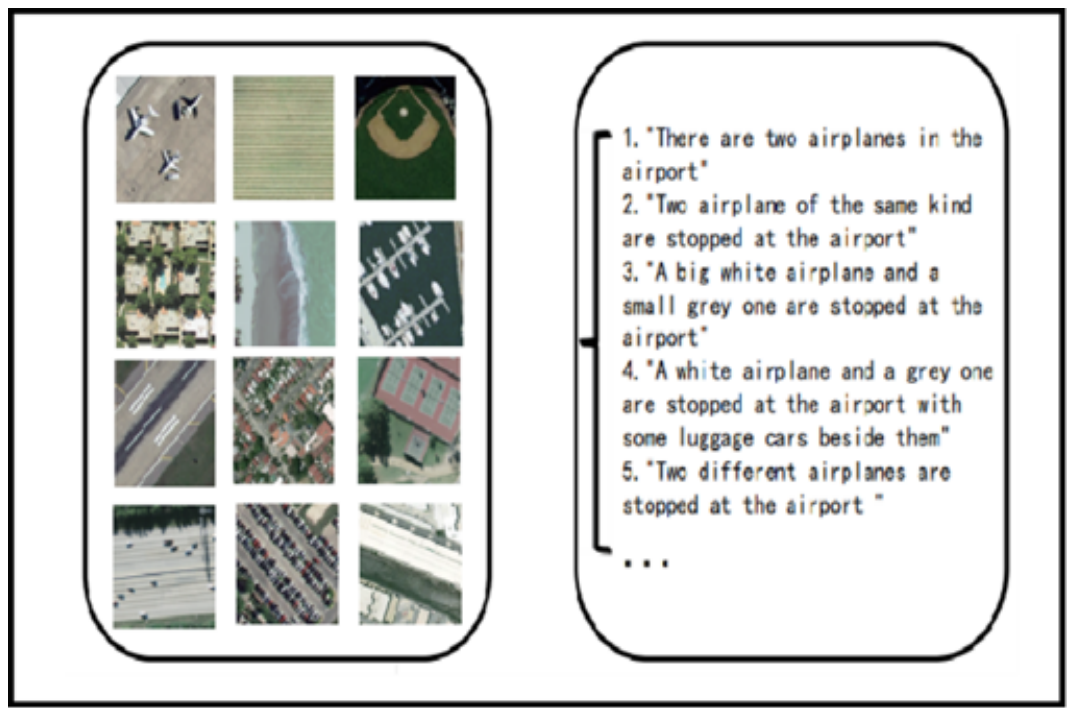

and the middle-level decreases the semantic gap among the high-level and low-level. Remote sensing semantic description mainly studies how to use precise and concise sentences to depict the specific content of the image. In order to fully express the semantic information in the image, this description task is generated. Its main purpose is to produce comprehensive sentences and summarize the semantic information of remote sensing images.

The whole process is to input a remote sensing image and automatically generate the representation of the image content by integrating the visual and text information of the remote sensing image with the computer. For this, Qu et al. attempts to interpret high-resolution images from the semantic perspective, and proposes a depth multimodal recurrent neural network model using visual information, which reflect image content and text information to describe object attributes, combined with the advantages

Figure 3. semantic segment Source: Panboonyuen et al., 2019

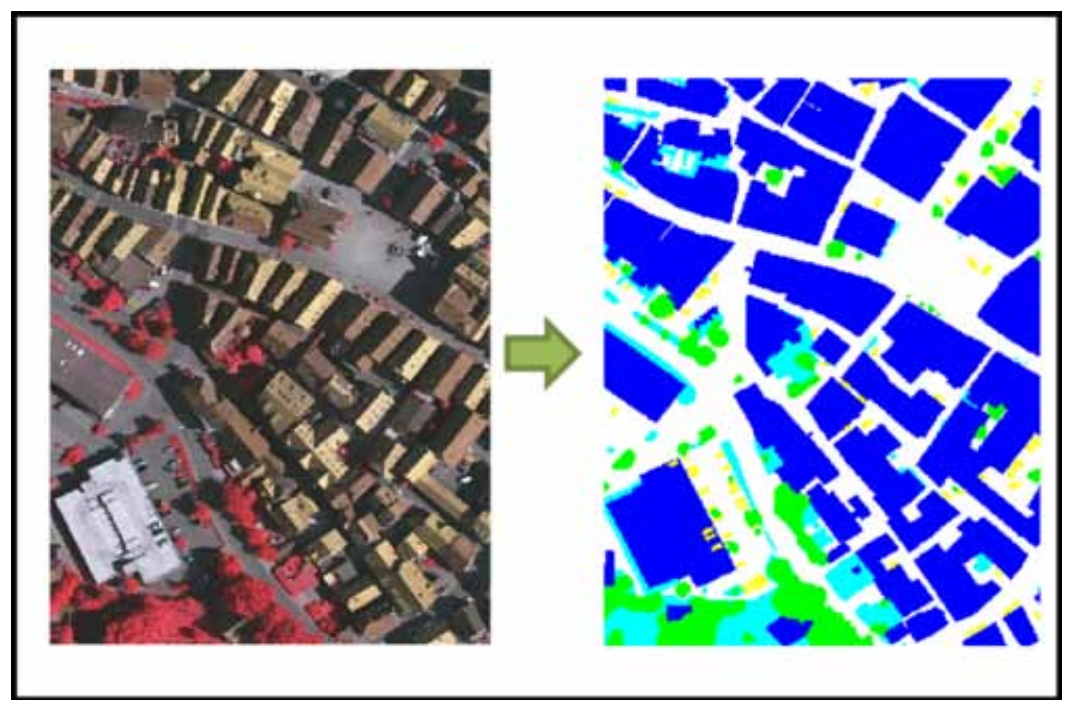




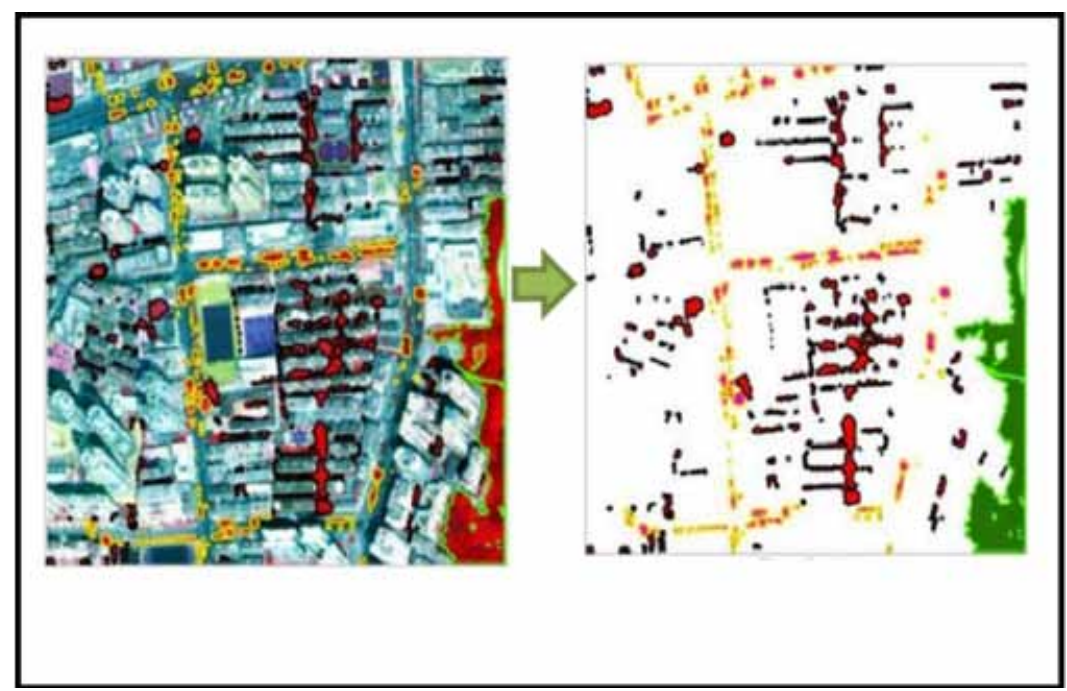

Figure 5. semantic search Source: Nys et al., 2019

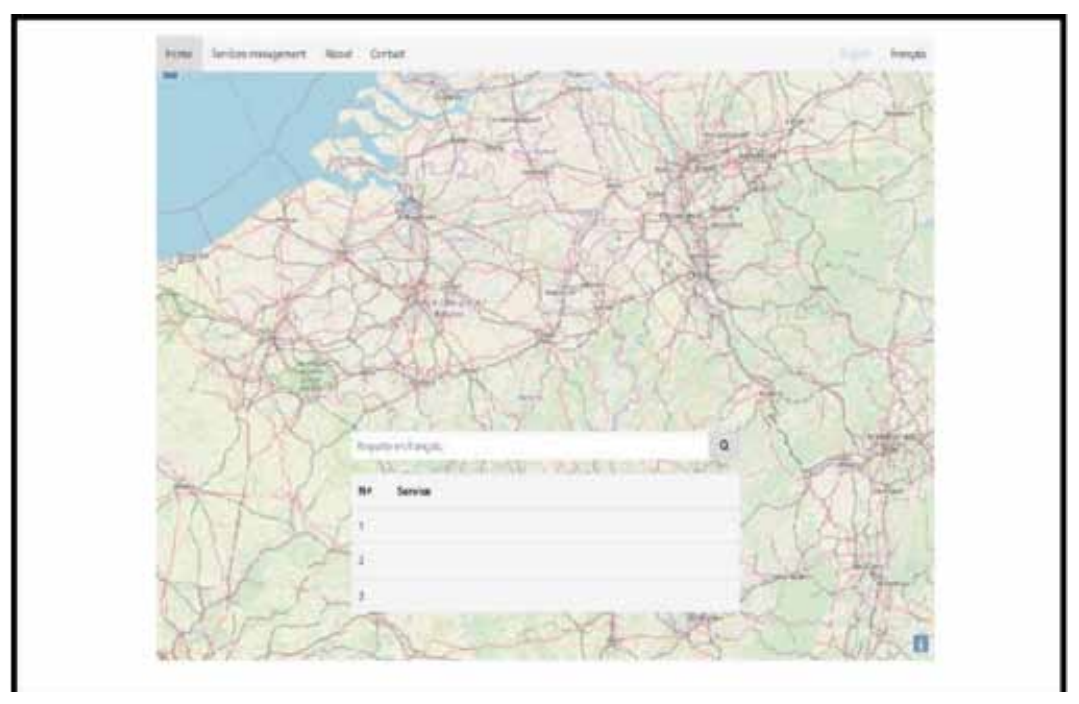

of the two to construct a high spatial resolution image semantic understanding model. This model combines different CNN and RNN or LSTM to achieve to distinguish the object attributes of the image and the relationship between objects (Qu et al., 2016). Zhan has carried out a series of researches on semantic modeling of remote sensing information based on ontology, and the construction of semantic description model (Zhan et al., 2008); Zhang has focused on the structural features of high-resolution remote sensing image space, scene space and se- mantic space based on deep learning, and realized the understanding and description of high-resolution remote sensing image content linked with natural language processing technology (X. Zhang et al., 2017). 
Figure 6. Semantic understanding and description of remote sensing images

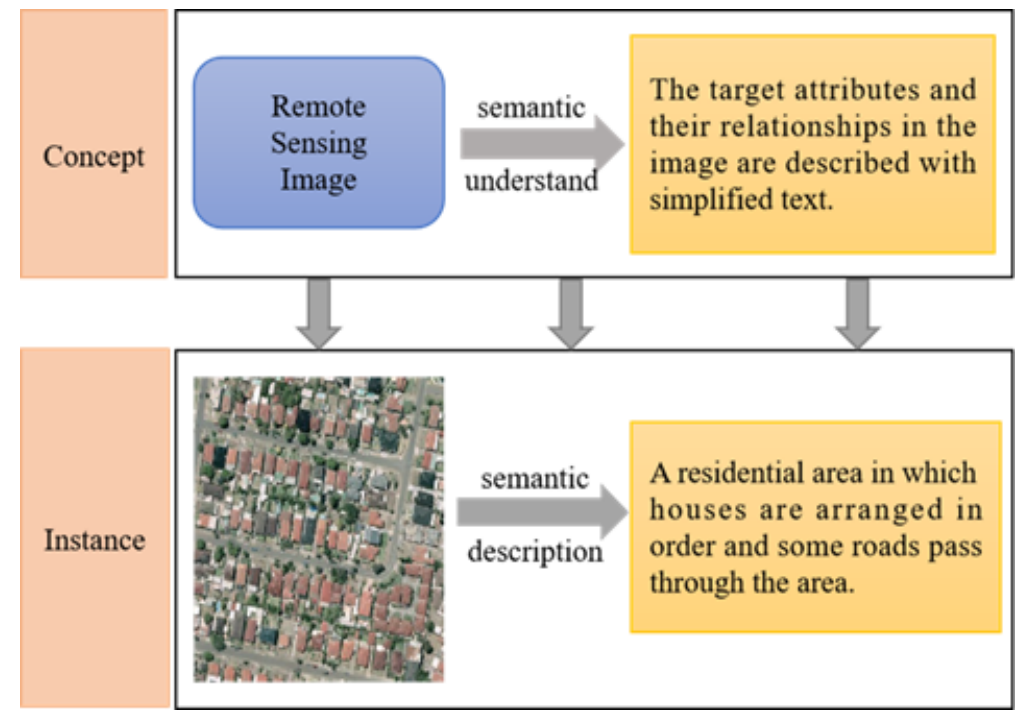

The understanding and description of remote sensing image content can provide decision-making support for remote sensing application, and has a broadly practical value. For instance, in the field of military investigation, the existing research algorithms can quickly identify important military targets such as ports, airports and ships from remote sensing images. The content comprehension and interpretation of remote sensing image can accurately and comprehensively understand the large-scale battlefield image, so as to get the real-time interpretation of battlefield geographical environment and dynamic intelligence generation. In civil use field, remote sensing image content understanding and description can provide easy to understand information such as disaster assessment, vegetation coverage, crop growth and urban change.

\subsection{Research and Application of Remote Sensing Semantic Segmentation}

In a broad sense, semantic segmentation of remote sensing image is an image classification task, which segments the semantic information of each pixel of remote sensing image. For segment the remote sensing image into regions with actual semantic information, the process of classifying the remote sensing image pixels according to the specified semantic criteria. Improvement of technical means, the method of semantic segmentation has also changed from traditional manual to it technology, that is, the use of computer professional software for semantic segmentation. In the wake of the emergence of high-resolution images, higher remote sensing segmentation techniques are emerging. Traditional machine learning algorithms such as random forest and SVM are broadly applied in remote sensing semantic segmentation.

With the flourish of artificial intelligence, semantic segmentation model based on deep learning has made great achievements. Aiming at the problems of multi-scale change of remote sensing image target, difficult segmentation of small target and noise interference, Hu structured a network model in view of double attention multi-scale feature fusion to improve the problem and effectively improve the segmentation accuracy. Panboonyuen et al. put forward a new CNN, which uses the attention mechanism with channel and the global convolution network of domain knowledge transfer to segment the semantics of multi object remote sensing satellite images (Panboonyuen et al., 2019); Morales et al. studied an automatic semantic segmentation method of remote sensing images taken by UAV (Morales et al., 2018). Boulila solves the difficulty of semantic segmentation of large remote sensing, and proposes a top-down method. The major purpose of the top level is to calculate 
Table 2. Common Data Sets of Remote Sensing Image Semantic Research

\begin{tabular}{|c|c|c|c|c|c|c|}
\hline Type & $\begin{array}{l}\text { Dataset } \\
\text { Name }\end{array}$ & $\begin{array}{l}\text { Dataset } \\
\text { Volume }\end{array}$ & Class & Characteristics & Source & Link \\
\hline \multirow{5}{*}{$\begin{array}{l}\text { Remote sensing } \\
\text { semantic } \\
\text { segmentation } \\
\text { dataset }\end{array}$} & $\begin{array}{l}\text { DOTA (Xia et } \\
\text { al., 2018) }\end{array}$ & 2806 & 15 & $\begin{array}{l}\text { The proportion of object } \\
\text { instances in aerial images } \\
\text { varies greatly. The fully } \\
\text { annotated image includes } \\
188282 \text { instances, each of } \\
\text { which is marked by an arbitrary } \\
\text { quadrilateral. }\end{array}$ & $\begin{array}{l}\text { Simultaneous } \\
\text { interpreting aerial } \\
\text { images from } \\
\text { distinct sensors } \\
\text { and platforms }\end{array}$ & $\begin{array}{l}\text { http://captain.whu.edu.cn/ } \\
\text { DOTAweb/dataset.html }\end{array}$ \\
\hline & $\begin{array}{l}\text { GID (GF-2) } \\
\text { (Tong et al., } \\
\text { 2018) }\end{array}$ & 150 & 15 & $\begin{array}{l}\text { GID images have intra class } \\
\text { diversity and inter class } \\
\text { separability; GF- } 2 \text { contains } \\
\text { panchromatic images with } \\
\text { spatial resolution of } 1 \mathrm{~m} \text { and } \\
\text { multispectral images of } 4 \mathrm{~m} .\end{array}$ & $\begin{array}{l}\text { From high- } \\
\text { definition earth } \\
\text { observation } \\
\text { system satellites }\end{array}$ & $\begin{array}{l}\text { http://captain.whu.edu. } \\
\text { cn/GID/ }\end{array}$ \\
\hline & $\begin{array}{l}\text { ISPRS } \\
\text { (Audebert et } \\
\text { al., 2017) }\end{array}$ & I & 6 & $\begin{array}{l}\text { Two state-of-the-art airborne } \\
\text { image data sets for urban sort } \\
\text { and 3D building reconstruction } \\
\text { test projects; } \\
\text { Digital terrain model generated } \\
\text { from high resolution orthogonal } \\
\text { photos and corresponding dense } \\
\text { image matching techniques. }\end{array}$ & $\begin{array}{l}\text { From airborne } \\
\text { sensors }\end{array}$ & $\begin{array}{l}\text { https://www2.isprs.org/ } \\
\text { commissions/comm3/wg4/ } \\
\text { semantic-labeling.html }\end{array}$ \\
\hline & $\begin{array}{l}\text { EvLab-SS } \\
\text { (M. Zhang et } \\
\text { al., 2017) }\end{array}$ & 35 & 11 & $\begin{array}{l}\text { Each image contains a complete } \\
\text { annotation of the geographic } \\
\text { survey. }\end{array}$ & $\begin{array}{l}\text { Produced by } \\
\text { the project of } \\
\text { geographical } \\
\text { condition survey } \\
\text { and mapping in } \\
\text { China }\end{array}$ & $\begin{array}{l}\text { http://earthvisionlab. } \\
\text { whu.edu.cn/zm/ } \\
\text { SemanticSegmentation/ } \\
\text { index.html }\end{array}$ \\
\hline & $\begin{array}{l}\text { SEN12MS } \\
\text { (Schmitt et al., } \\
\text { 2019) }\end{array}$ & 180748 & 3 & $\begin{array}{l}\text { It consists of sentinel-1 } \\
\text { dual polarization SAR data, } \\
\text { sentinel- } 2 \text { multispectral image } \\
\text { and MODIS land cover map. }\end{array}$ & $\begin{array}{l}\text { Created by the } \\
\text { tum organization }\end{array}$ & $\begin{array}{l}\text { https://mediatum.ub.tum. } \\
\text { de/1474000 }\end{array}$ \\
\hline \multirow{3}{*}{$\begin{array}{l}\text { Semantic } \\
\text { classification } \\
\text { dataset }\end{array}$} & $\begin{array}{l}\text { RSSCN7 } \\
\text { (Zou et al., } \\
2015 \text { ) }\end{array}$ & 2800 & 7 & $\begin{array}{l}\text { The scene images taken in } \\
\text { different seasons and different } \\
\text { weather conditions have wide } \\
\text { diversity, and samples are taken } \\
\text { at different scales. }\end{array}$ & $\begin{array}{l}\text { Some are from } \\
\text { Google Earth, } \\
\text { others are } \\
\text { collected by the } \\
\text { author }\end{array}$ & $\begin{array}{l}\text { https://sites.google.com/site/ } \\
\text { qinzoucn/documents }\end{array}$ \\
\hline & $\begin{array}{l}\text { SIRI-WHU } \\
\text { (B. Zhao, } \\
\text { 2015, 2016; } \\
\text { Q. Zhu et al., } \\
\text { 2016) }\end{array}$ & 2400 & 12 & $\begin{array}{l}\text { Covering urban areas in China, } \\
\text { the spatial resolution of each } \\
\text { image is } 2 \text { meters. }\end{array}$ & $\begin{array}{l}\text { Get from Google } \\
\text { Earth }\end{array}$ & $\begin{array}{l}\text { http://www.lmars.whu.edu. } \\
\text { cn/prof_web/zhongyanfei/e- } \\
\text { code.html }\end{array}$ \\
\hline & $\begin{array}{l}\text { RSD46-WHU } \\
\text { (Long et al., } \\
\text { 2017; Xiao et } \\
\text { al., 2017) }\end{array}$ & 138000 & 46 & $\begin{array}{l}\text { It is used for remote sensing } \\
\text { image scene classification; } \\
\text { Large scale opening up. }\end{array}$ & $\begin{array}{l}\text { Collected by } \\
\text { Google Earth and } \\
\text { Tianditu }\end{array}$ & $\begin{array}{l}\text { http://www.lmars.whu.edu. } \\
\text { cn/prof_web/xiaozhifeng/ } \\
\text { dataset.html }\end{array}$ \\
\hline \multirow[b]{2}{*}{$\begin{array}{l}\text { Semantic image } \\
\text { search dataset }\end{array}$} & $\begin{array}{l}\text { PatternNet } \\
\text { (Chaudhuri et } \\
\text { al., 2017; } \\
\text { Shao et al., } \\
\text { 2018) }\end{array}$ & 30400 & 38 & $\begin{array}{l}\text { For remote sensing image } \\
\text { search; Large scale high- } \\
\text { resolution image data; For } \\
\text { American cities. }\end{array}$ & $\begin{array}{l}\text { Collect from } \\
\text { Google Earth or } \\
\text { google map API }\end{array}$ & $\begin{array}{l}\text { https://sites.google.com/ } \\
\text { view/zhouwx/dataset }\end{array}$ \\
\hline & $\begin{array}{l}\text { DLRSD } \\
\text { (Shao et al., } \\
\text { 2018; Shao, } \\
2020 \text { ) }\end{array}$ & 2100 & 21 & $\begin{array}{l}\text { Dense label dataset for multi } \\
\text { label tasks. }\end{array}$ & $\begin{array}{l}\text { Proposed by } \\
\text { National Key } \\
\text { Laboratory of } \\
\text { Surveying and } \\
\text { mapping and } \\
\text { Remote Sensing } \\
\text { Information } \\
\text { Engineering }\end{array}$ & $\begin{array}{l}\text { https://drive.google.com/ } \\
\text { file/d/1DtKiauowCB0ykjFe } \\
\text { 8v0OVvT76rEfOk0v/view }\end{array}$ \\
\hline
\end{tabular}


the object features extracted from the remote sensing image, and the target at the bottom level is to ascertain the class of each pixel applying the information calculated in the upper level (Boulila, 2019). Besides, in the semantic segmentation task, the academic community has constantly proposed semantic segmentation network models, such as full convolutional neural network (FCN) (Y. Zhao et al., 2019), SegNet (Badrinarayanan et al., 2017), Deconvolution Network (J. Fu et al., 2020), DeepLab (Niu et al., 2018) and U-Net (Y. Fu et al., 2019) etc., and each network has made different improvements to different problems.

At present, remote sensing image segmentation technology has been widely used in disaster monitoring, meteorological modeling, urban planning, road extraction and so on. Lebedev et al. studied the application of remote sensing image semantic segmentation in the prediction of near precipitation, and proposed a precipitation near prediction system based on satellite image. The precipitation field was reconstructed by using satellite data, and the prediction precipitation model was used for the near prediction. The research expanded the coverage and provided the basis for global near prediction (Lebedev et al., 2019). Hordiiuk et al. applied remote sensing image se- mantic segmentation to ship monitoring, and identified ships through optical remote sensing image, and proposed a convolution neural network method to increase the detection accuracy and shorten the measurement time (Hordiiuk et al., 2019). Shi applied semantic segmentation of remote sensing images to road extraction research. Aiming at the problems of complex background features and no obvious difference in categories of high-resolution remote sensing images, a way for extracting urban trunk roads from optical remote sensing images was proposed. It is mainly combined with spectral space classification and local measured homogeneity to extract the initial road network, and then based on the road characteristics, eliminate false roads, and finally extract the road network generated by local weighted regression algorithm, so as to achieve road extraction (Shi et al., 2013).

\subsection{Research and Application of Remote Sensing Semantic Classification}

Remote sensing semantic classification simply refers to the semantic classification according to the way of human perception of high-level image semantics. For a long time, researchers in this field have also made significant achievements. Aiming at the semantic gap between low-level visual features and image high-level semantics, Zhang et al. proposed a visual bag-of-words model (bow) to produce intermediate level functions, and combined with the object semantic classification algorithm of optimal segmentation scale, the semantic classification of remote sensing images was better realized (J. Zhang et al., 2016). It is to be observed that scene semantic sort is regarded as the key of image semantic classification. Remote sensing scene classification is the result of global interpretation and analysis of the scene semantic contents of remote sensing image. The remote sensing image is divided into corresponding categories by obtaining the scene semantic information of remote sensing image. Figure 7 shows the flow of scene classification. Zou et al. put forward a study of image scene classification based on deep learning, reinterpreted the difficulty of feature selection as the problem of feature reconstruction, and selected the reconfigurable feature as the distinguishing feature to achieve good feature selection effect (Zou et al., 2015). At the same time, a new benchmark dataset is provided to promote the field classification of images. Liu et al. put forward a random scaling convolution neural network for high-resolution remote sensing image scene sort (Y. Liu et al., 2016). Chen et al. focused on the remote sensing image scene classification method in view of deep learning. The emergence of deep learning can obtain higher-level semantic information, thus more accurate expression of remote sensing image scene (L. Chen et al., 2019).

Semantic classification is widely used in urban, forestry and other fields. Wen et al. applied ultrahigh-resolution remote sensing images to the semantic classification of urban trees, and proposed a new pixel object patch three-tier framework. According to human cognition and conceptualization, urban trees are classified into Park, roadside and residential institutional trees (Wen et al., 2017). Du et al. applied high-resolution remote sensing images with GIS to the semantic classification of buildings (Du et al., 2015). The purpose of semantic classification of architectural objects is to use 
Figure 7. Process chart of scene semantic classification of remote sensing image

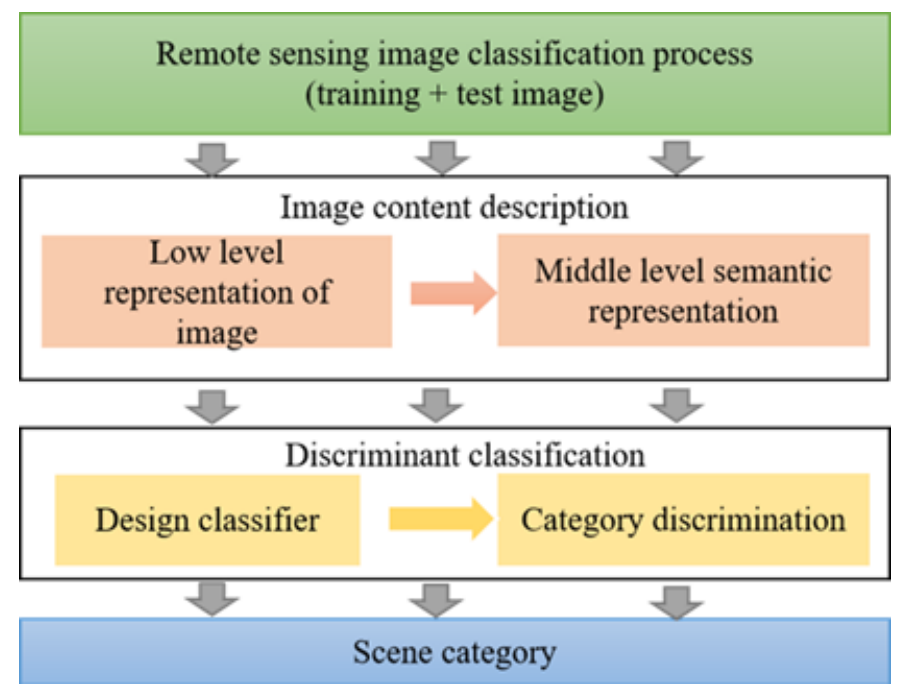

a group of semantic categories recognized and conceptualized by people to mark buildings. A largescale layer constrained by GIS data is proposed to generate a single image object, while a small-scale layer provides a two-level segmentation mechanism and a semi supervised method for the component features within the object. According to the characteristics of random forest that can handle massive samples and high-dimensional and heterogeneous characteristics, different semantic sort of buildings at different levels is realized.

\subsection{Research and Application of Remote Sensing Semantic Retrieval}

Currently, the research of remote sensing semantic retrieval is mainly applied in remote sensing image search. The progress of remote sensing semantic search technology in the field of remote sensing satellite image retrieval provides more accurate and more in line with the needs of the public. Foreign image retrieval technology is more mature, semantic retrieval technology, mainly reflected in the use of semantic web technology for image semantic retrieval. For example, Nys et al. developed a semantic search system for remote sensing web platform in order to narrow the semantic gap between users unfamiliar with remote sensing vocabulary and remote sensing service supplier. The system uses natural language algorithm, thesaurus and knowledge map to support semantic retrieval (Nys et al., 2019). Kumar et al. put forward a semantic classifier technology in the current semantic web environment. The technology is mainly based on image content retrieval, integrating grid computing and advanced database concepts, which effectively improves the efficiency of remote sensing image retrieval (Kumar et al., 2018).

Domestic related research started late, but has also made great achievements. Liu et al. put forward a remote sensing image semantic retrieval mean in view of deep information represented by correlation rules, which has the traditional low-level visual properties (J. Liu \& Liu, 2015). It is based on the principle that the spatial distribution patterns of pixels are reflected by the association rules of remote sensing images. When two images include certain identical or similar objects, the spatial distribution patterns of the two images are similar. Song et al. give a novel deep hash CNN, which redefined the traditional image retrieval problem as image visual and semantic retrieval. Combined with deep hash learning, similar images can be retrieved in the same framework and their semantic tags can be classified at the same time. Moreover, the framework can use the semantic tags of returned 
Table 3. Research and Application Comparison of Remote Sensing Semantic Construction

\begin{tabular}{|l|l|l|l|}
\hline Semantic Construction & Research Methods & Limitations & Application Fields \\
\hline $\begin{array}{l}\text { Semantic understanding and } \\
\text { description of high-resolution } \\
\text { remote sensing images }\end{array}$ & $\begin{array}{l}\text { Machine learning, } \\
\text { statistical learning, data } \\
\text { mining, deep learning. }\end{array}$ & $\begin{array}{l}\text { Single data, lack of } \\
\text { accuracy, GPU resource } \\
\text { occupation is too large. }\end{array}$ & $\begin{array}{l}\text { Crop growth, disaster } \\
\text { assessment, urban } \\
\text { planning, military target } \\
\text { recognition, etc. }\end{array}$ \\
\hline Semantic segmentation & $\begin{array}{l}\text { Random forest, support } \\
\text { vector machine, CNN, } \\
\text { FCN, etc. }\end{array}$ & $\begin{array}{l}\text { The segmentation model } \\
\text { has too many parameters, } \\
\text { time-consuming } \\
\text { calculation, insufficient } \\
\text { segmentation accuracy and } \\
\text { insufficient sample size. }\end{array}$ & $\begin{array}{l}\text { Precipitation forecast, } \\
\text { disaster relief, urban } \\
\text { planning, traffic } \\
\text { management, etc. }\end{array}$ \\
\hline Semantic classification & $\begin{array}{l}\text { Optical image } \\
\text { classification method, } \\
\text { remote sensing image } \\
\text { feature information sort, } \\
\text { deep learning. }\end{array}$ & $\begin{array}{l}\text { The accuracy of } \\
\text { feature extraction } \\
\text { and classification is } \\
\text { insufficient. }\end{array}$ & $\begin{array}{l}\text { Natural disaster } \\
\text { monitoring, land use, } \\
\text { urban planning, vegetation } \\
\text { mapping, etc. }\end{array}$ \\
\hline Semantic search & $\begin{array}{l}\text { Semantic Web } \\
\text { technology, ontology } \\
\text { technology, machine } \\
\text { learning. }\end{array}$ & $\begin{array}{l}\text { Remote sensing image } \\
\text { information mining ability } \\
\text { is insufficient, search } \\
\text { accuracy is not high. }\end{array}$ & $\begin{array}{l}\text { Environmental monitoring, } \\
\text { disaster analysis, urban } \\
\text { planning, land use, } \\
\text { military fields. }\end{array}$ \\
\hline
\end{tabular}

similar images, which can better explore the database and play an important role in retrieving images from databases with only a few real samples (Song et al., 2019).

\section{DISCUSSION}

As shown in Table 3, this section mainly makes a comprehensive comparison on the semantic research methods and application fields of remote sensing data in Section 3. Then, the characteristics of the current research field are discussed respectively according to the content of the table, so as to grasp the problems and challenges of the current semantic-based remote sensing data processing technology. More importantly, it provides new research ideas and provides exploration direction for researchers in the next step.

As can be seen from Table 3, there are abundant research methods in the four directions of semantic construction, including not only the traditional machine learning methods, but also the current mainstream deep learning methods. What is more noteworthy is that the application field has been expanded more and more. It is mainly reflected in meteorology, environment, agriculture, transportation, urban planning, disaster assessment, target recognition and so on. However, it is inevitable that there are some limitations in each research field. Specifically, for the research of remote sensing semantic understanding, the single research data leads to the problem of low accuracy. There are many researches on semantic segmentation model, but there are still some problems, such as too much calculation and too much parameters, which lead to low segmentation efficiency. At the same time, it is urgent to improve the classification accuracy and search accuracy. Therefore, how to enrich the sample size, optimize the research model, upgrade the research methods, so as to improve the accuracy and efficiency of the results are the key problems to be solved.

\subsection{Challenges and Application Prospects of Remote Sensing Semantic Understanding and Description}

At present, the high-resolution remote sensing image understanding methods have basically completed the transition from pixel level classification to object-oriented classification method, but its 


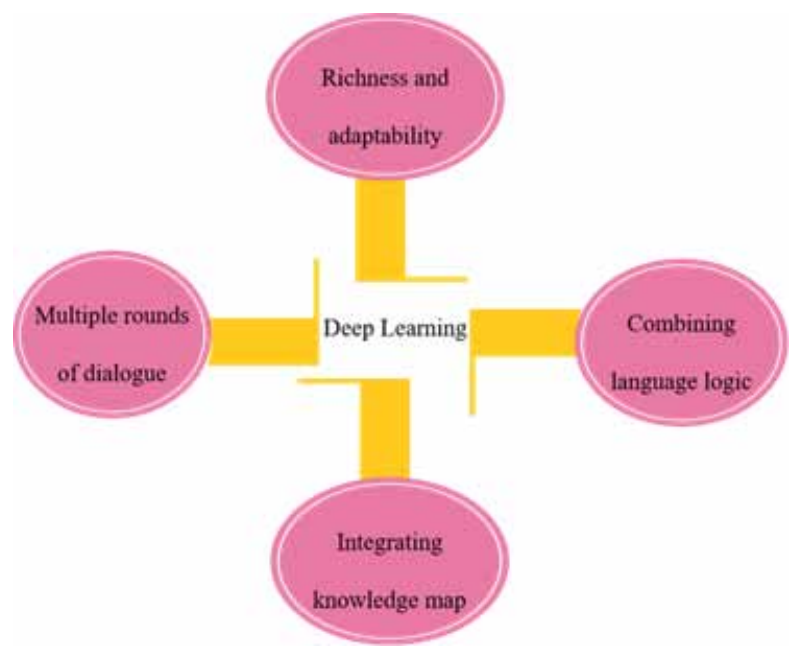

interpretation level is only to the ground object category level, without reasoning and understanding of the scene. These studies only identify the object in the image or obtain the class label of the image, while ignoring the object attributes and the relationship between each object. In the research of highresolution remote sensing image from the semantic level, there are still various deficiencies, such as single data, insufficient database richness, algorithm is not advanced, and takes up too much memory resources, which makes the semantic understanding and description of remote sensing image unilateral and inaccurate. Therefore, there is a lot of space to improve the database. In terms of computer vision, image description is a very important research field, but in remote sensing fields, remote sensing image description has certain complexity. At present, more researchers need to pay more attention to the semantic description of remote sensing image, enrich the remote sensing description data set, consider the characteristics of remote sensing image, combine with deep learning technology, fully mine the meaning of remote sensing image, so as to obtain the remote sensing semantic scene description in line with practical application, which will play a major role in many industries.

Based on the current research situation, in order to better apply deep learning to remote sensing image semantic understanding, the key points of the summary in Figure 8 should be combined. What needs to be explained is that the richness of semantics is mainly manifested in the multi-dimensional semantic representation and the cross meaning of the semantic itself; self-adaptation is reflected in the ability to correctly express the corresponding semantic understanding for different situations. However, for each key point, it is the difficulty of technical research, which needs researchers to conduct in-depth study.

\subsection{Challenges and Application Prospects of Remote Sensing Semantic Segmentation}

The current remote sensing semantic segmentation methods have strong generalization ability. Random Forest, SVM and CNN are used in semantic segmentation task, but they do not make the best of hierarchical model information and spatial semantic information, so they cannot obtain better semantic segmentation accuracy. And there are still some problems in the segmentation, such as the target space is scattered, the scale is variable and the background is complex. On this basis, FCN performs end-to-end semantic segmentation, reduces the amount of computation, improves the accuracy of semantic segmentation, and thus produces a series of networks. However, there are still some problems such as too many parameters of segmentation model, time-consuming calculation and 
insufficient segmentation accuracy, which cannot fully meet the current large-scale remote sensing image semantic segmentation assignment. There is still a lot of room to increase the accuracy of semantic segmentation. Therefore, how to combine the characteristics of remote sensing image, apply deep convolution neural network to remote sensing image segmentation task, and design a good performance remote sensing image segmentation model is still a big difficulty to challenge.

At present, the research work in remote sensing image segmentation field in case segmentation is not like a huge image case segmentation research work in computer vision field, that is, the number of objects of interest and the specific location of targets cannot be identified in detail. This is worthy of further study in the future. Moreover, the difficulty of obtaining large-scale data sets and the complexity of data annotation methods lead to the difficulty of successful application of neural networks based on deep learning of small samples. Moreover, most of the current researchers use supervised deep learning algorithm, and need a large number of accurate data annotation, timeconsuming and labor-consuming. Therefore, the study of unsupervised deep learning algorithm for semantic segmentation will become an important direction in the future.

\subsection{Challenges and Application Prospects of Remote Sensing Semantic Classification}

In Section 3, the authors discuss that the semantic sort of remote sensing images is mainly reflected in the scene semantic classification. Therefore, this section mainly discusses the challenges and application prospects of scene semantic classification. At present, a lot of research work has been done, mainly through the direct use of optical image classification methods and remote sensing image feature information classification. However, relying on the traditional classification methods, it still cannot get a good classification effect in terms of the expression of remote sensing image feature information and classification accuracy. On this basis, researchers introduce deep learning into it to settle the problem of image feature extraction effectively. However, thanks to the features of remote sensing big data, the research on semantic feature extraction and classification of remote sensing images is still in the initial stage. Building a deep learning network model to learn more high-level and rich scene semantic information of remote sensing images is the main research direction in the future.

\subsection{Challenges and Application Prospects of Remote Sensing Semantic Retrieval}

Compared with the traditional retrieval based on text and image content, semantic retrieval can accurately describe the image object and help users understand. Semantic retrieval technology provides a new solution for image retrieval, and shows good application effect. However, in the face of massive and complex remote sensing images, users' demand will increase rapidly. It is an important issue that the researchers must face and urgently need to solve for more effective remote sensing image retrieval technology so as to quickly obtain the images that users in different industries are interested in. Compared with the current amount of remote sensing image data, the utilization rate of remote sensing image is still very low, far below people's expectations, which is due to the complexity of remote sensing itself, industry barriers, and the lack of information mining ability of remote sensing data.

At present and in the future, in order to provide users with high-quality remote sensing image data quickly and efficiently, effective semantic retrieval algorithm should be studied, and new remote sensing image retrieval technology should be developed, so that further promote researchers to carry out research on specific applications, thus accelerating the improvement of information mining ability of remote sensing data, which has an important role in promoting the utilization of remote sensing images. 


\section{CONCLUSION}

In this paper, through the investigation and discussion of remote sensing image semantic construction technology and application research, mainly for semantic understanding, semantic segmentation, semantic classification and semantic search four research areas are carefully analyzed and discussed. It can be seen that the current remote sensing image data processing combined with computer software and hardware technology makes remote sensing data in the field of semantic construction has made many important achievements It is widely used in the fields of people's livelihood and military affairs. Moreover, in the current information age environment, advanced applications and services need more knowledge with clear semantics than information, and acquiring semantic knowledge needs semantic technology support. With the explosive growth of remote sensing data and the changing needs of the times, the demand for semantic technology is growing. However, the current research on semantic technology of remote sensing image big data is still facing great challenges. Such as the fusion algorithm of multi-source remote sensing data information, the deep learning method of remote sensing data semantic construction, and the remote sensing big data mining technology based on knowledge driven. Remote sensing image semantic research is a developing research field. In the future, new computer technology will be integrated to promote the establishment of remote sensing image semantic construction model, which will be applied in different fields. More new applications and application methods need to be further studied and explored by the majority of researchers.

\section{ACKNOWLEDGMENT}

This work was supported in part by the Key Laboratory Foundation of National Defence Technology under Grant 61424010208, Science and Technology Project of SGCC "Research on Feature Recognition and Prediction of Typical Ice and Wind Disaster for Transmission Lines Based on Small Sample Machine Learning Method" (No. 4501223825), National Natural Science Foundation of China (No. 41911530242, 41975142, 41875184), 5150 Spring Specialists (05492018012, 05762018039), Major Program of the National Social Science Fund of China (Grant No.17ZDA092), 333 High-Level Talent Cultivation Project of Jiangsu Province (BRA2018332), Royal Society of Edinburgh, UK and China Natural Science Foundation Council (RSE Reference: 62967_Liu_2018_2) under their Joint International Projects funding scheme, Innovation Team of "Six Talent Peaks" in Jiangsu Province (Grant No.TD-XYDXX-004) and basic Research Programs (Natural Science Foundation) of Jiangsu Province (BK20191398, BK20180794). 


\section{REFERENCES}

Audebert, N., Le Saux, B., \& Lefèvrey, S. (2017, March). Fusion of heterogeneous data in convolutional networks for urban semantic labeling. In 2017 Joint Urban Remote Sensing Event (JURSE) (pp. 1-4). IEEE. doi:10.1109/ JURSE.2017.7924566

Badrinarayanan, V., Kendall, A., \& Cipolla, R. (2017). Segnet: A deep convolutional encoder-decoder architecture for image segmentation. IEEE Transactions on Pattern Analysis and Machine Intelligence, 39(12), 2481-2495. doi:10.1109/TPAMI.2016.2644615 PMID:28060704

Boulila, W. (2019). A top-down approach for semantic segmentation of big remote sensing images. Earth Science Informatics, 12(3), 295-306. doi:10.1007/s12145-018-00376-7

Chaudhuri, B., Demir, B., Chaudhuri, S., \& Bruzzone, L. (2017). Multilabel remote sensing image retrieval using a semisupervised graph-theoretic method. IEEE Transactions on Geoscience and Remote Sensing, 56(2), 1144-1158. doi:10.1109/TGRS.2017.2760909

Chen, L., Cui, X., Li, Z., Yuan, Z., Xing, J., Xing, X., \& Jia, Z. (2019). A New Deep Learning Algorithm for SAR Scene Classification Based on Spatial Statistical Modeling and Features Re-Calibration. Sensors (Basel), 19(11), 2479. doi:10.3390/s19112479 PMID:31151259

Chen, Y., Zhang, T., Feng, X., Bai, T., \& Ma, J. (2012). Research on agricultural information retrieval model based on double semantic space. Xinjiang Nongye Daxиe Xиеbao, 35(3), 253-258.

Collarana, D., Galkin, M., Traverso-Ribón, I., Lange, C., Vidal, M. E., \& Auer, S. (2017). Semantic data integration for knowledge graph construction at query time. In 2017 IEEE 11th International Conference on Semantic Computing (ICSC) (pp. 109-116). IEEE. doi:10.1109/ICSC.2017.85

Dai, F., Zhai, X., Shi, G.-q., \& Du, C.-y. (2018). Modeling ontological knowledge network for aerospace equipment development. Journal of Zhejiang University (Engineering Science), 52(10), 2023-2034.

Du, S., Zhang, F., \& Zhang, X. (2015). Semantic classification of urban buildings combining vhr image and gis data: An improved random forest approach. ISPRS Journal of Photogrammetry and Remote Sensing, 105(jul), 107-119. doi:10.1016/j.isprsjprs.2015.03.011

Fu, J., Liu, J., Li, Y., Bao, Y., Yan, W., Fang, Z., \& Lu, H. (2020). Contextual deconvolution network for semantic segmentation. Pattern Recognition, 101, 107152. doi:10.1016/j.patcog.2019.107152

Fu, Y., Liu, K., Shen, Z., Deng, J., Gan, M., Liu, X., Lu, D., \& Wang, K. (2019). Mapping impervious surfaces in town-rural transition belts using China's GF-2 imagery and object-based deep CNNs. Remote Sensing, 11(3), 280. doi: $10.3390 / \mathrm{rs} 11030280$

Garcia, D. C. F., Gattaz, C. C., \& Cruvinel, P. E. (2019, January). Information Retrieval: A Case Study on Contributions of Greimasian Semiotics to Semantic Computing in Agriculture for Knowledge Management. In 2019 IEEE 13th International Conference on Semantic Computing (ICSC) (pp. 478-484). IEEE.

Gardent, C., \& Kallmeyer, L. (2003, April). Semantic construction in F-TAG. 10th conference of the European Chapter of the Association for Computational Linguistics.

Ghazouani, F., Farah, I. R., \& Solaiman, B. (2019). A multi-level semantic scene interpretation strategy for change interpretation in remote sensing imagery. IEEE Transactions on Geoscience and Remote Sensing, 57(11), 8775-8795. doi:10.1109/TGRS.2019.2922908

Hordiiuk, D., Oliinyk, I., Hnatushenko, V., \& Maksymov, K. (2019, April). Semantic Segmentation for Ships Detection from Satellite Imagery. In 2019 IEEE 39th International Conference on Electronics and Nanotechnology (ELNANO) (pp. 454-457). IEEE. doi:10.1109/ELNANO.2019.8783822

Jadhav, J. K., \& Singh, R. (2018). Automatic semantic segmentation and classification of remote sensing data for agriculture. Mathematical Models in Engineering, 4(2), 112-137. doi:10.21595/mme.2018.19840

Jiang, Y., Han, T., \& Wang, D. (2018, March). The investigation of identifying method on grass fire by FY-3 VIRR images. In MIPPR 2017: Remote Sensing Image Processing, Geographic Information Systems, and Other Applications (Vol. 10611). International Society for Optics and Photonics. 
Kasimov, D. R., Kuchuganov, V. N., \& Kuchuganov, A. V. (2019). Principles of organization of the strategies of content-based analysis of aerospace images. In International conference on intelligent information technologies for industry (pp. 289-299). Springer.

Khamparia, A., \& Pandey, B. (2017). Comprehensive analysis of semantic web reasoners and tools: A survey. Education and Information Technologies, 22(6), 3121-3145. doi:10.1007/s10639-017-9574-5

Kim, S., Lee, W., Park, Y. S., Lee, H. W., \& Lee, Y. T. (2016, December). Forest fire monitoring system based on aerial image. In 20163 rd international conference on information and communication technologies for disaster management (ICT-DM) (pp. 1-6). IEEE. doi:10.1109/ICT-DM.2016.7857214

Klusch, M., Kapahnke, P., Schulte, S., Lecue, F., \& Bernstein, A. (2016). Semantic web service search: A brief survey. KI-Künstliche Intelligenz, 30(2), 139-147. doi:10.1007/s13218-015-0415-7

Kumar, N. S., Arun, M., \& Dangi, M. K. (2018). Remote sensing image retrieval using object-based, semantic classifer techniques. International Journal of Information and Communication Technology, 13(1), 68-82. doi:10.1504/IJICT.2018.090432

Lebedev, V., Ivashkin, V., Rudenko, I., Ganshin, A., Molchanov, A., Ovcharenko, S., \& Solomentsev, D. et al. (2019, July). Precipitation nowcasting with satellite imagery. In Proceedings of the 25th ACM SIGKDD International Conference on Knowledge Discovery \& Data Mining (pp. 2680-2688). doi:10.1145/3292500.3330762

Liu, C. W., Wu, T. H., Tsai, M. H., \& Kang, S. C. (2018). Image-based semantic construction reconstruction. Automation in Construction, 90, 67-78. doi:10.1016/j.autcon.2018.02.016

Liu, J., \& Liu, S. (2015, July). Semantic retrieval for remote sensing images using association rules mining. In 2015 IEEE International Geoscience and Remote Sensing Symposium (IGARSS) (pp. 509-512). IEEE. doi:10.1109/IGARSS.2015.7325812

Liu, Y., Zhong, Y., Fei, F., \& Zhang, L. (2016, July). Scene semantic classification based on random-scale stretched convolutional neural network for high-spatial resolution remote sensing imagery. In 2016 IEEE international geoscience and remote sensing symposium (IGARSS) (pp. 763-766). IEEE. doi:10.1109/IGARSS.2016.7729192

Long, Y., Gong, Y., Xiao, Z., \& Liu, Q. (2017). Accurate object localization in remote sensing images based on convolutional neural networks. IEEE Transactions on Geoscience and Remote Sensing, 55(5), 2486-2498. doi:10.1109/TGRS.2016.2645610

Mahmood, K., Takahashi, H., \& Alobaidi, M. (2015, March). A semantic approach for traceability link recovery in aerospace requirements management system. In 2015 IEEE Twelfth International Symposium on Autonomous Decentralized Systems (pp. 217-222). IEEE. doi:10.1109/ISADS.2015.33

Morales, G., Kemper, G., Sevillano, G., Arteaga, D., Ortega, I., \& Telles, J. (2018). Automatic segmentation of Mauritia exuosa in unmanned aerial vehicle (uav) imagery using deep learning. Forests, 9(12), 736. doi:10.3390/ f9120736

Niu, Z., Liu, W., Zhao, J., \& Jiang, G. (2018). Deeplab-based spatial feature extraction for hyperspectral image classification. IEEE Geoscience and Remote Sensing Letters, 6(2), 251-255. doi:10.1109/LGRS.2018.2871507

Nys, G. A., Kasprzyk, J. P., Hallot, P., \& Billen, R. (2019). A Semantic Retrieval System for Remote Sensing Web Platforms. The International Archives of the Photogrammetry, Remote Sensing and Spatial Information Sciences, 42(W13), 1593-1599. doi:10.5194/isprs-archives-XLII-2-W13-1593-2019

Panboonyuen, T., Jitkajornwanich, K., Lawawirojwong, S., Srestasathiern, P., \& Vateekul, P. (2019). Semantic segmentation on remotely sensed images using an enhanced global convolutional network with channel attention and domain specific transfer learning. Remote Sensing, 11(1), 83. doi:10.3390/rs11010083

Pauwels, P., Zhang, S., \& Lee, Y. C. (2017). Semantic web technologies in AEC industry: A literature overview. Automation in Construction, 73, 145-165. doi:10.1016/j.autcon.2016.10.003

Phiri, D., \& Morgenroth, J. (2017). Developments in landsat land cover classification methods: A review. Remote Sensing, 9(9), 967. doi:10.3390/rs9090967

Qu, B., Li, X., Tao, D., \& Lu, X. (2016, July). Deep semantic understanding of high resolution remote sensing image. In 2016 International Conference on Computer, Information and Telecommunication Systems (CITS) (pp. 1-5). IEEE. doi:10.1109/CITS.2016.7546397 
Ren, L., Meng, Z., Wang, X., Zhang, L., \& Yang, L. T. (2020). A data-driven approach of product quality prediction for complex production systems. IEEE Transactions on Industrial Informatics, 1. Advance online publication. doi:10.1109/TII.2020.3001054

Schmitt, M., Hughes, L. H., Qiu, C., \& Zhu, X. X. (2019). Sen12ms-a curated dataset of georeferenced multispectral sentinel-1/2 imagery for deep learning and data fusion. arXiv preprint arXiv:1906.07789.

Shao, Z., Yang, K., \& Zhou, W. (2018). Performance evaluation of single-label and multi-label remote sensing image retrieval using a dense labeling dataset. Remote Sensing, 10(6), 964. doi:10.3390/rs10060964

Shao, Z., Zhou, W., Deng, X., Zhang, M., \& Cheng, Q. (2020). Multilabel Remote Sensing Image Retrieval Based on Fully Convolutional Network. IEEE Journal of Selected Topics in Applied Earth Observations and Remote Sensing, 13, 318-328. doi:10.1109/JSTARS.2019.2961634

Shi, W., Miao, Z., \& Debayle, J. (2013). An integrated method for urban main-road centerline extraction from optical remotely sensed imagery. IEEE Transactions on Geoscience and Remote Sensing, 52(6), 3359-3372. doi:10.1109/TGRS.2013.2272593

Song, W., Li, S., \& Benediktsson, J. A. (2020). Deep hashing learning for visual and semantic retrieval of remote sensing images. IEEE Transactions on Geoscience and Remote Sensing, 1-12. doi:10.1109/TGRS.2020.3035676

Taymaz, H. M., Özyer, T., \& Cangelir, C. (2013, August). A knowledge-based semantic tool for standard part management in aerospace industry. In 2013 IEEE 14th International Conference on Information Reuse \& Integration (IRI) (pp. 634-642). IEEE. doi:10.1109/IRI.2013.6642528

Tong, X. Y., Xia, G. S., Lu, Q., Shen, H., Li, S., You, S., \& Zhang, L. (2018). Learning transferable deep models for land-use classification with high-resolution remote sensing images. arXiv preprint arXiv:1807.05713.

Wang, S., Zhang, L., Zhang, Y., Sun, J., Pang, C., Tian, G., \& Cao, N. (2018). Natural language semantic construction based on cloud database. Comput. Mater. Continua, 57(3), 603-619. doi:10.32604/cmc.2018.03884

Wang, X., Yang, L. T., Song, L., Wang, H., Ren, L., \& Deen, J. (2020). A tensor-based multi- attributes visual feature recognition method for industrial intelligence. IEEE Transactions on Industrial Informatics. Advance online publication. doi:10.1109/TII.2020.2999901

Wang, X., Yang, L. T., Wang, Y., Ren, L., \& Deen, M. J. (2020). ADTT: A highly-efficient distributed tensortrain decomposition method for IIoT big data. IEEE Transactions on Industrial Informatics. Advance online publication. doi:10.1109/TII.2020.2967768

Wen, D., Huang, X., Liu, H., Liao, W., \& Zhang, L. (2017). Semantic classification of urban trees using very high resolution satellite imagery. IEEE Journal of Selected Topics in Applied Earth Observations and Remote Sensing, 10(4), 1413-1424. doi:10.1109/JSTARS.2016.2645798

Xia, G. S., Bai, X., Ding, J., Zhu, Z., Belongie, S., Luo, J., \& Zhang, L. et al. (2018). DOTA: A large-scale dataset for object detection in aerial images. In Proceedings of the IEEE Conference on Computer Vision and Pattern Recognition (pp. 3974-3983). doi:10.1109/CVPR.2018.00418

Xiao, Z., Long, Y., Li, D., Wei, C., Tang, G., \& Liu, J. (2017). High-resolution remote sensing image retrieval based on cnns from a dimensional perspective. Remote Sensing, 9(7), 725. doi:10.3390/rs9070725

Xiong, X., Angal, A., Wu, A., Wang, Z., Barnes, W., \& Salomonson, V. (2017, July). 15 years of aqua MODIS on-orbit operation, calibration, and performance. In 2017 IEEE International Geoscience and Remote Sensing Symposium (IGARSS) (pp. 4695-4698). IEEE. doi:10.1109/IGARSS.2017.8128050

Xu, X., Shen, B., Yin, X., Khosravi, M. R., Wu, H., Qi, L., \& Wan, S. (2020). Edge Server Quantification and Placement for Offloading Social Media Services in Industrial Cognitive IoV. IEEE Transactions on Industrial Informatics. Advance online publication. doi:10.1109/TII.2020.2987994

Yuan, L. Y., \& Wang, X. C. (2014). Survey of semantics for internet of things. Computer.

Zhan, Q., Zhang, X., \& Li, D. (2008). Ontology-based semantic description model for discovery and retrieval of geospatial information. The International Archives of the Photogrammetry, Remote Sensing and Spatial Information Sciences, 32(B4). 
Zhang, J., Li, T., Lu, X., \& Cheng, Z. (2016). Semantic classification of high-resolution remote-sensing images based on mid-level features. IEEE Journal of Selected Topics in Applied Earth Observations and Remote Sensing, 9(6), 2343-2353. doi:10.1109/JSTARS.2016.2536943

Zhang, M., Hu, X., Zhao, L., Lv, Y., Luo, M., \& Pang, S. (2017). Learning dual multi-scale manifold ranking for semantic segmentation of high-resolution images. Remote Sensing, 9(5), 500. doi:10.3390/rs9050500

Zhang, X., Li, X., An, J., Gao, L., Hou, B., \& Li, C. (2017, July). Natural language description of remote sensing images based on deep learning. In 2017 IEEE International Geoscience and Remote Sensing Symposium (IGARSS) (pp. 4798-4801). IEEE. doi:10.1109/IGARSS.2017.8128075

Zhao, B., Zhong, Y., Xia, G. S., \& Zhang, L. (2015). Dirichlet-derived multiple topic scene classification model for high spatial resolution remote sensing imagery. IEEE Transactions on Geoscience and Remote Sensing, 54(4), 2108-2123. doi:10.1109/TGRS.2015.2496185

Zhao, B., Zhong, Y., Zhang, L., \& Huang, B. (2016). The Fisher kernel coding framework for high spatial resolution scene classification. Remote Sensing, 8(2), 157. doi:10.3390/rs8020157

Zhao, Y., Zhang, Y., Yi, K., \& Lei, Y. (2019, July). Understanding and Improving the Intermediate Features of FCN in Semantic Segmentation. In 2019 International Conference on Communications, Information System and Computer Engineering (CISCE) (pp. 415-419). IEEE. doi:10.1109/CISCE.2019.00098

Zhong, F. J., Gu, L. C., \& Zhang, Y. H. (2009). Survey on applications of semantic web. Computer Engineering and Design.

Zhu, J., Shi, Q., Chen, F., Shi, X., Do, Z., \& Qin, Q. (2016). Research status and development trends of remote sensing big data. Journal of Image and Graphics, 21(11), 1425-1439.

Zhu, Q., Zhong, Y., Zhao, B., Xia, G. S., \& Zhang, L. (2016). Bag-of-visual-words scene classifier with local and global features for high spatial resolution remote sensing imagery. IEEE Geoscience and Remote Sensing Letters, 13(6), 747-751. doi:10.1109/LGRS.2015.2513443

Zou, Q., Ni, L., Zhang, T., \& Wang, Q. (2015). Deep learning based feature selection for remote sensing scene classification. IEEE Geoscience and Remote Sensing Letters, 12(11), 2321-2325. doi:10.1109/ LGRS.2015.2475299

Hui Lu received the B.S. degree in Computer Science and Technology from BoHai University, China in 2019, and is currently studying in software engineering of Nanjing University of Information Technology. Her research interests include semantic segmentation of remote sensing images in building extraction.

Qi Liu (M'11, SM'18) received the B.S. degree in Computer Science and Technology from Zhuzhou Institute of Technology, China in 2003, and M.S. and Ph.D. in Data Telecommunications and Networks from the University of Salford, UK in 2006 and 2010. His research interests include context awareness, data communication in MANET and WSN, and smart grid. His recent research work focuses on intelligent agriculture and meteorological observation systems based on WSN.

Xiaodong Liu (M'00, SM'17) received his PhD in Computer Science from De Montfort University and joined Napier in 1999. He is a professor and is currently leading the Intelligence-Driven Smart Systems research group in the School of Computing, Edinburgh Napier University. He was the director of Centre for Information \& Software Systems. He is an active researcher in software engineering and Al with internationally excellent reputation and leading expertise in context-aware adaptive services, service evolution, mobile clouds, pervasive computing, smart systems, and semantic data modelling.

Yonghong Zhang is with the School of Automation, Nanjing University of Information Science and Technology. He mainly engaged in Precision Instruments and Machinery, Pattern Recognition and Intelligent Systems, Remote Sensing Information Processing, as well as Higher Education Teaching Management and Research Work. He has led multiple major research projects of the National Natural Science Foundation of China (NSFC). 